October, 2002

IFT-P.077/02

hep-th/0210154

LPTHE-02.52

\title{
Integrability and Conformal Symmetry in Higher Dimensions: A Model with Exact Hopfion Solutions
}

\author{
O. Babelon ${ }^{1}$ and L. A. Ferreira ${ }^{2}$ \\ (1) Laboratoire de Physique Theorique et Hautes Energies \\ Universités Paris VI - Paris VII \\ Boîte 126, Tour 16, $1^{\mathrm{er}}$ étage \\ 4 Place Jussieu, F-75252, Paris CEDEX 05, France \\ (2) Instituto de Física Teórica - IFT/UNESP \\ Rua Pamplona 145 \\ 01405-900 São Paulo-SP, Brazil
}

\begin{abstract}
We use ideas on integrability in higher dimensions to define Lorentz invariant field theories with an infinite number of local conserved currents. The models considered have a two dimensional target space. Requiring the existence of Lagrangean and the stability of static solutions singles out a class of models which have an additional conformal symmetry. That is used to explain the existence of an ansatz leading to solutions with non trivial Hopf charges.
\end{abstract}




\section{Introduction}

We consider in this paper Lorentz invariant local field theories in a space-time of $d+1$ dimensions, with an infinite number of local conserved currents. In order to construct such models we use the generalized zero curvature conditions in any dimension proposed in [1]. The basic ingredient of that approach is to take the potentials of the zero curvature to lie in non-semisimple Lie algebras with an infinite dimensional abelian ideal. Restricting ourselves to theories with two dimensional target space, we obtain those algebraic structures using a $s l(2)$ algebra realized in terms of differential opera-

tors on two parameters (Schwinger construction) and taking the ideal as the space of functions of these parameters.

The equations of motion of the theories we obtain from the generalized zero curvature are of the form

$$
\partial^{\mu} \mathcal{K}_{\mu}=0 \quad \partial^{\mu} u \mathcal{K}_{\mu}=0 \quad \partial^{\mu} u^{*} \mathcal{K}_{\mu}-\partial^{\mu} u \mathcal{K}_{\mu}^{*}=0
$$

where $u$ is a complex scalar field parametrizing our two-dimensional target space, and $\mathcal{K}_{\mu}$ is a complex function of the fields $u$ and $u^{*}$ and their derivatives. These theories have an infinite number of local conserved currents given by

$$
J_{\mu}=\mathcal{K}_{\mu} \frac{\delta G}{\delta u}-\mathcal{K}_{\mu}^{*} \frac{\delta G}{\delta u *}
$$

with $G$ being any functional of the fields $u$ and $u^{*}$ but not of their derivatives.

Since eqs.(1.1) are five real equations for two real fields, we have a constrained system. However, there is a class of functionals $\mathcal{K}_{\mu}$ that automatically satisfies the constraints. They are given by

$$
\mathcal{K}_{\mu}=\mathcal{F} h_{\mu \nu} \partial^{\nu} u, \quad h_{\mu \nu}=\partial_{\mu} u \partial_{\nu} u^{*}-\partial_{\mu} u^{*} \partial_{\nu} u
$$

where $\mathcal{F}$ is a real function of $u, u^{*}$, and their first order derivatives. The above quantity solves automaticaly the last two equations in (1.1).

We show that the only unconstrained theories that can be derived from an action principle are those where the Lagrangean is a function of $h^{2} / f^{2}$ only, with $f$ being a real function of $u$ and $u^{*}$ and $h^{2}=h_{\mu \nu} h^{\mu \nu}$. For these theories the currents above are Noether currents corresponding to the invariance of the action under area preserving diffeomorphisms on target space equipped with an area form $d u \wedge d u^{*} / f$.

In order to have static solution we have to overcome Derrick's theorem. Since $h^{2} / f^{2}$ scales as $\lambda^{-4} h^{2} / f^{2}$ under the scaling transfomation $x^{\mu} \rightarrow \lambda x^{\mu}$, we consider the 
Lagrangeans

$$
\mathcal{L}=\left(\frac{h^{2}}{2 f^{2}}\right)^{d / 4}
$$

which give scale invariant static energy in $d$ space dimensions.

Such scale invariance has led us to study more closely the symmetry group of the equations of motion. We found that the space-time and target space symmetries always commute. We found that in the static case, the equations of motion have the full conformal symmetry group $O(d+1,1)$. In the non-static case only the scale transformation is a symmetry of the equations of motion, besides the usual Poincaré group. However, that scale transfomation is not a symmetry of the action. In target space, we found that besides the area preserving diffeomorphisms there is one extra symmetry of the full equations of motion, which however is not a symmetry of the Lagrangean either. We can however combine that with the space-time scale transformation to build a true symmetry of the action and then obtain an extra Noether current.

Applying S. Lie's ideas about symmetries of differential equations to the conformal symmetry group, we are immediatly led to construct educated ansatz for solutions of the equations of motion. For the theory (1.3) with $d=3$, we used the $O(4,1)$ conformal symmetry to introduced two cyclic variables (angles) and then reduce the static equations of motion to an ordinary differential equation for a profile function. We explain in this way the origin of the ansatz used in [2] to construct the exact hopfion solutions with non-trivial Hopf charges.

The charge associated to the extra Noether current, coming from the mixing of target space and space-time scale transformations, is not conserved due to boundary terms. Its rate of change in time is in fact the static energy. We have evaluated the flux of that current for the case of the 3-dimensional hopfion solutions and found that the leaking of the charge takes place along the $z$-axis, where that flux is singular. This implies that the hopfion solutions carry a line structure with them associated to such line of singularity of the Noether current. This may be relevant in the scatterring process of those solutions.

\section{Zero curvature on loop spaces}

One of the basic ingredients of two-dimensional integrable models is the so-called LaxZakharov-Shabat zero curvature condition

$$
\partial_{\mu} B_{\nu}-\partial_{\nu} B_{\mu}+\left[B_{\mu}, B_{\nu}\right]=0 \quad \mu, \nu=0,1
$$


It is the starting point for several methods for constructing exact solutions, like the dressing, inverse scattering and Riemmann-Hilbert methods. In addition, in $1+1$ dimensions the relation (2.4) is a conservation law, since it is a sufficient condition for the path ordered integral

$$
W(\Gamma)=P_{\Gamma} \exp \int_{\Gamma} B_{\mu} d x^{\mu}
$$

to be independent of the path $\Gamma$, as long as the end points are kept fixed. Consequently, on a closed loop one has that $W$ is a constant and so can be set to unit. Therefore, by choosing appropriate boundary conditions at space infinity (or taking space to be a circle and so space-time a cylinder) one gets that the quantities

$$
Q_{N} \equiv \operatorname{Tr}\left(P \exp \int_{\text {space }} B_{x} d x\right)^{N}
$$

are conserved in time. These are the conservation laws responsible for the integrability of a two dimensional theory possessing the zero curvature representation (2.4).

Under these considerations it is then natural to try to generalize the Lax-ZakharovShabat equation (2.4) to theories defined on a space-time of dimension $d+1$ as the sufficient condition for ordered integrals of a generalized connection on a $d$ dimensional surface to be surface independent. Similar reasonings and appropriate boundary conditions should then lead to conservation laws. That is exactly the idea put foward in [1]. The equivalent of (2.5), in $d+1$ dimensions, would be a $d$ dimensional surface ordered integral of a rank $d$ antisymmetric tensor $B_{\mu_{1} \ldots \mu_{d}}$.

An important issue in the implementation of such ideas is the ordering of the integration on the hypersurfaces. Given a $d$-dimensional surface $\Sigma$ with border $\partial \Sigma$, we choose a base point $x_{0}$ in $\partial \Sigma$ and then scan $\Sigma$ with $(d-1)$-dimensional closed surfaces containing $x_{0}$. Such closed surfaces are ordered and labeled by a parameter $\sigma_{d}$ such that, for instance, $\sigma_{d}=0$ correspond to the infinitesimally small surface around $x_{0}$, and $\sigma_{d}=2 \pi$ to $\partial \Sigma$. Then each one of these surfaces are scanned in their turn by $(d-2)$-dimensional closed surfaces with base point $x_{0}$ too, and labeled by a parameter $\sigma_{d-1}$. The process goes on until we reach two dimensional surfaces which are scanned by ordinary closed loops starting and ending at $x_{0}$.

These ideas find their best formulation, in fact, in generalized loop space. Given a $d+1$ dimensional space time $M$, one considers the space $\Omega^{(d-1)}\left(M, x_{0}\right)$ of $d-1$ closed surfaces in $M$ with a fixed base point $x_{0}$. A path in $\Omega^{(d-1)}\left(M, x_{0}\right)$ is a $d$ dimensional surface in $M$. Therefore, the conserved charges can be obtained from path ordered integrals in the generalized loop space, in a similar way as charges in $1+1$ dimensions are obtained from path ordered integral in space-time (see (2.6)). Indeed, the two approaches become the same in $1+1$ dimensions, since for $d=1$ the loop space 
coincides with space-time. In addition, as we show below, the rank $d$ antisymmetric tensor $B_{\mu_{1} \ldots \mu_{d}}$ can be used to construct a one-form connection in generalized loop space by integrating it on a generalized loop $((d-1)$-dimensional closed surface).

Notice that in the scanning just described we have a nested structure of generalized loop spaces, since the closed $(d-n)$-dimensional surfaces have base point $x_{0}$ and so belong to $\Omega^{(d-n)}\left(M, x_{0}\right)$. We can then introduce, in the space-time $M$, a set of antisymmetric tensors of rank varying from 1 to $d$ and use them to construct one-form connections on each one of those loop spaces.

The zero curvature conditions for such loop space connections usually leads to highly non-local expressions in space-time. In order to make things as local as possible, it was found in [1] that it is advantageous to parallel transport quantities in a given loop space using the connections in the lower loop spaces. Of course we do not want things to depend upon the way we scan the surfaces. It then follows that all those connections on loop spaces will have to be flat to guarantee the scanning independence. Therefore, we have a nested structure of zero curvatures involving those antisymmetric tensors. However, only the loop space connection associated to the the rank $d$ tensor leads to conserved charges in $d+1$ dimensions. We refer to [1] for a more detailed explanation of the construction of such connections.

In the applications of the present paper we only consider the highest and lowest tensors, i.e. a rank $d$ antisymmetric tensor $B_{\mu_{1} \ldots \mu_{d}}$, and a vector $A_{\mu}$. In the scanning of the $d$-dimensional surface $\Sigma_{d}$, each one of its points belong to just one $(d-1)$ dimensional surface $\Sigma_{d-1}$. Similarly, that point of $\Sigma_{d}$ lying in $\Sigma_{d-1}$ belongs to only one $(d-2)$-dimensional closed surface $\Sigma_{d-2}$ scanning $\Sigma_{d-1}$. Repeating this procedure we see that each point of $\Sigma_{d}$ belongs to just one 1-dimensional loop of the scanning. We then use that loop and $A_{\mu}$ to parallel transport $B_{\mu_{1} \ldots \mu_{d}}$ at that point of $\Sigma_{d}$ back to the base point $x_{0}$.

The surface $\Sigma_{d}$ is a path in the generalized loop space $\Omega^{(d-1)}$. Therefore, the ordered integral of the rank $d$ antisymmetric tensor $B_{\mu_{1} \ldots \mu_{d}}$ over $\Sigma_{d}$, can be seen as a path ordered integral in $\Omega^{(d-1)}$ of a one-form connection. We define that one-form on the generalized loop space $\Omega^{(d-1)}$ by the formula

$$
\mathcal{A}=\int_{0}^{2 \pi} d \sigma_{1} \ldots d \sigma_{d-1} W^{-1} B_{\mu_{1} \ldots \mu_{d}} W \frac{d x^{\mu_{1}}}{d \sigma_{1}} \ldots \frac{d x^{\mu_{d-1}}}{d \sigma_{d-1}} \delta x^{\mu_{d}}
$$

where $\sigma_{i}, i=1, \ldots d-1$, parametrize the closed $d-1$ dimensional surface in $M$, according to the scanning described above. $W$ is built as the path ordered integral of the one-form connection $A_{\mu}$, in a similar way as in (2.5). However, in order for things to be independent of the scanning we take the connection $A_{\mu}$ to be flat. It then follows 
that $W$ is path independent, and so it is uniquely defined on every point of $\Sigma_{d}$ once its value in $x_{0}$ is given. Therefore, the quantity $W^{-1} B_{\mu_{1} \ldots \mu_{d}} W$, is local in $\Sigma_{d}$. The variation $\delta x^{\mu}$ corresponds to infinitesimal changes in the closed $(d-1)$-dimensional surface $\Sigma_{d-1}$, and it is in fact what characterizes $\mathcal{A}$ as a one-form in $\Omega^{(d-1)}$. Therefore, the condition for the ordered integral on a $d$ dimensional surface

$$
\mathcal{P} \exp \left(\int_{\Sigma_{d}} d \sigma_{1} \ldots d \sigma_{d} W^{-1} B_{\mu_{1} \ldots \mu_{d}} W \frac{d x^{\mu_{1}}}{d \sigma_{1}} \ldots \frac{d x^{\mu_{d}}}{d \sigma_{d}}\right)
$$

to be surface independent, is that the one-form $\mathcal{A}$ should be flat, i.e.

$$
\mathcal{C}=\delta \mathcal{A}+\mathcal{A} \wedge \mathcal{A}=0
$$

That is the generalization of the Lax-Zakharov-Shabat equation (2.4) proposed in [1], for theories defined on a space-time $M$ of $d+1$ dimensions. Under appropriate boundary conditions it leads to the conserved charges

$$
\operatorname{Tr}\left(\mathcal{P} \exp \left(\int_{\text {space }} d \sigma_{1} \ldots d \sigma_{d} W^{-1} B_{\mu_{1} \ldots \mu_{d}} W \frac{d x^{\mu_{1}}}{d \sigma_{1}} \ldots \frac{d x^{\mu_{d}}}{d \sigma_{d}}\right)\right)^{N}
$$

Notice that for $d=1$, one recovers the usual two-dimensional Lax-Zakharov-Shabat zero curvature condition.

Clearly, the condition (2.9) is still non-local in $M$, since $\mathcal{A}$ is a multidimensional integral there. To get zero curvature representations of the equations of motion of local field theories in the space-time $M$, we make special choice for $B_{\mu_{1} \ldots \mu_{d}}$ and $A_{\mu}$. It was shown in (2.4) that if one takes $A_{\mu}$ to live on a non-semisimple Lie algebra and $B_{\mu_{1} \ldots \mu_{d}}$ to belong to the corresponding abelian ideal then the local equations

$$
d B+A \wedge B=0 \quad F=d A+A \wedge A=0
$$

are sufficient conditions for the vanishing of the curvature (2.9) in loop space. The second condition in (2.11) is imposed to have things independent of the way one scans the integrated surfaces. It implies that $A_{\mu}$ is obtained from $W$ as a pure gauge connection, i.e.

$$
A_{\mu}=-\partial_{\mu} W W^{-1}
$$

We shall then take (2.11) as our generalized zero curvature conditions. Introducing the dual of $B$

$$
\tilde{B}_{\mu} \equiv \frac{1}{d} \epsilon_{\mu \mu_{1} \ldots \mu_{d}} B^{\mu_{1} \ldots \mu_{d}}
$$

one gets that the first relation in (2.11) becomes

$$
D^{\mu} \tilde{B}_{\mu}=\partial^{\mu} \tilde{B}_{\mu}+\left[A^{\mu}, \tilde{B}_{\mu}\right]=0
$$


As a consequence of such relation it follows that the conserved charges (2.10) are now obtained from local conserved currents

$$
J_{\mu} \equiv W^{-1} \tilde{B}_{\mu} W \quad \rightarrow \quad \partial^{\mu} J_{\mu}=0
$$

The number of conserved currents is therefore equal to the dimension of the abelian ideal where $B$ lives.

Notice that the equations (2.11) are sufficient local conditions for the vanishing of the curvature (2.9), in any generalized loop of $\Omega^{(d-1)}$. However, if one is not interested in conserved charges one can restrict to the case of infinitesimal loops only. In such case there are weaker local sufficient conditions for the vanishing of the curvature (2.9). In the $2+1$ dimensional case for instance, those conditions are given by

$$
\omega=0 \quad \nu(B)=0
$$

with

$$
\omega=d B+A \wedge B \quad \nu(\cdot)=[d A+A \wedge A-B, \cdot]
$$

In addition, one gets the Bianchi identities

$$
d \nu(\cdot)+A \wedge \nu(\cdot)=-[\omega, \cdot] \quad d \omega+A \wedge \omega=\nu(B)
$$

The two-form $\nu$ is called the fake curvature in the mathematical literature [3]. Of course, (2.11) together with the fact $B$ is abelian imply (2.16).

\section{The $\lambda-\bar{\lambda}$ realization of $\operatorname{sl}(2)$}

We now consider field theories such that the equations of motion are written as the local zero curvature conditions (2.11). Since, the potential $B$ has to live on an abelian ideal we consider a Poincaré type non-semisimple Lie algebra $\mathcal{G}=T+P$, satisfying

$$
[T, T] \subset T \quad[T, P] \subset P \quad[P, P]=0
$$

where $T$ is a Lie algebra and $P$ a representation of it. In order for the model to be integrable we need an infinite number of conserved currents of the form (2.15), and consequently the representation $P$ has to be infinite dimensional. In order to get that, we shall use the Schwinger's construction. Let $R(T)$ be a (finite) matrix representation of the algebra $T$, i.e.

$$
\left[R(T), R\left(T^{\prime}\right)\right]=R\left(\left[T, T^{\prime}\right]\right)
$$


Consider a number of oscillators equal to the dimension of $R$,

$$
\left[a_{i}, a_{j}\right]=0 \quad\left[a_{i}^{\dagger}, a_{j}^{\dagger}\right]=0 \quad\left[a_{i}, a_{j}^{\dagger}\right]=\delta_{i j} \quad i, j=1, \ldots \operatorname{dim} R
$$

Then, it follows that the operators

$$
S(T) \equiv \sum_{i, j} a_{i}^{\dagger} R_{i j}(T) a_{j}
$$

constitute a representation of $T$

$$
\left[S(T), S\left(T^{\prime}\right)\right]=S\left(\left[T, T^{\prime}\right]\right)
$$

The oscillators can be realized in terms of differential operators on some parameters $\lambda_{i}$, as $a_{i} \equiv \frac{\partial}{\partial \lambda_{i}}$ and $a_{i}^{\dagger}=\lambda_{i}$.

In the case of the $\operatorname{sl}(2)$ algebra, namely

$$
\left[T_{3}, T_{ \pm}\right]= \pm T_{ \pm} ; \quad\left[T_{+}, T_{-}\right]=2 T_{3}
$$

one gets that its two dimensional matrix representation leads to the following realization in terms of differential operators

$$
T_{+} \equiv \lambda \frac{d}{d \bar{\lambda}} ; \quad T_{-} \equiv \bar{\lambda} \frac{d}{d \lambda} ; \quad T_{3} \equiv \frac{1}{2}\left(\lambda \frac{d}{d \lambda}-\bar{\lambda} \frac{d}{d \bar{\lambda}}\right)
$$

The states of the representations corresponding to such realization are functions of $\lambda$ and $\bar{\lambda}$. The action of the operators are given by

$$
\begin{aligned}
T_{3} \lambda^{p} \bar{\lambda}^{q} & =\frac{p-q}{2} \lambda^{p} \bar{\lambda}^{q} \\
T_{+} \lambda^{p} \bar{\lambda}^{q} & =q \lambda^{p+1} \bar{\lambda}^{q-1} \\
T_{-} \lambda^{p} \bar{\lambda}^{q} & =p \lambda^{p-1} \bar{\lambda}^{q+1}
\end{aligned}
$$

Notice that from (3.26) the action of $T_{3}, T_{ \pm}$leaves the sum of the powers of $\lambda$ and $\bar{\lambda}$ invariant. Therefore, one can construct irreducible representations by considering the states

$$
|(p, q), m\rangle \equiv \lambda^{p+m} \bar{\lambda}^{q-m}
$$

with $m \in \mathbb{Z}$ and $(p, q)$ being any pair of numbers (real or even complex). Then

$$
\begin{aligned}
& T_{3}|(p, q), m\rangle=\left(\frac{p-q}{2}+m\right)|(p, q), m\rangle \\
& T_{+}|(p, q), m\rangle=(q-m)|(p, q), m+1\rangle \\
& T_{-}|(p, q), m\rangle=(p+m)|(p, q), m-1\rangle
\end{aligned}
$$


On the subspace with fixed $(p+q)$, the Casimir operator acts as:

$$
\left(T_{3}^{2}+\frac{1}{2}\left(T_{+} T_{-}+T_{-} T_{+}\right)\right)|(p, q), m\rangle=s(s+1)|(p, q), m\rangle, \quad s=\frac{1}{2}(p+q)
$$

The parameter $s$ is the spin of the representation.

From the relations (3.28) one notices that if $p$ is an integer, then $|(p, q),-p\rangle$ is a lowest weight state, since it is annihilated by $T_{-}$. Analogously, if $q$ is an integer, then $|(p, q), q\rangle$ is a highest weight state. If $p$ and $q$ are integers and $q>-p$, then the irrep. is finite dimensional. In order to have integer spin representations we need $\frac{p-q}{2} \in \mathbb{Z}$. The spin zero state is $\left|(p, q),-\frac{p-q}{2}\right\rangle=(\lambda \bar{\lambda})^{\frac{p+q}{2}}$. Notice however, that not all irreps. for integer spin will have the zero spin state. The reason is that if $p$ is a negative integer, or $q$ is a positive integer, then the representation will truncate before reaching the zero spin state.

\section{The local zero curvature for theories with two scalar fields}

We shall consider field theories in a $d+1$-dimensional space-time and two dimensional target space. So, the fields are Lorentz scalars taking values on the sphere $S^{2}$, torus $T^{2}$, the plane $R^{2}$, etc. We shall parametrize them by a complex scalar field $u$. We introduce the equations of motion through the local zero curvature conditions (2.11). We take $A_{\mu}$ to be a flat connection as in (2.12) with the group element $W$ being given in the two dimensional representation by

$$
W=\frac{1}{\sqrt{1+|u|^{2}}}\left(\begin{array}{cc}
1 & i u \\
i u^{*} & 1
\end{array}\right)
$$

Such element can be written in any representation as

$$
W \equiv e^{i u T_{+}} e^{\varphi T_{3}} e^{i u^{*} T_{-}} ; \quad \varphi \equiv \log \left(1+|u|^{2}\right)
$$

Then, using the realization (3.25) one gets

$$
A_{\mu} \equiv \frac{1}{1+|u|^{2}}\left(-i \partial_{\mu} u \lambda \frac{d}{d \bar{\lambda}}-i \partial_{\mu} u^{*} \bar{\lambda} \frac{d}{d \lambda}+\left(u \partial_{\mu} u^{*}-u^{*} \partial_{\mu} u\right) \frac{1}{2}\left(\lambda \frac{d}{d \lambda}-\bar{\lambda} \frac{d}{d \bar{\lambda}}\right)\right)
$$

The equations of motions come from the first relation in (2.11) (or equivalently (2.14)), with the dual potential (2.13) being given by

$$
\tilde{B}_{\mu}^{(s)} \equiv \frac{1}{1+|u|^{2}}\left(\mathcal{K}_{\mu} \lambda^{s+1} \bar{\lambda}^{s-1}-\mathcal{K}_{\mu}^{*} \lambda^{s-1} \bar{\lambda}^{s+1}\right)
$$


The vector $\mathcal{K}_{\mu}$ is a priori a functional of $u, u^{*}$ and their derivatives. Notice that we have chosen $B_{\mu}^{(s)}$ to live in a representation where $p=q=s$, and it has components only in the direction of the states with eigenvalues \pm 1 of $T_{3}$. The factor $1 /\left(1+|u|^{2}\right)$ was introduced for convenience since it could be absorbed into the definition of $\mathcal{K}_{\mu}$.

Plugging (4.31) and (4.32) into (2.14) one gets terms in the direction of states with with spins (eigenvalues of $T_{3}$ ) $0, \pm 1$ and \pm 2 . The terms with spins -1 and -2 are complex conjugate of those with spins 1 and 2 respectively. The equations we get are those given in (1.1), together with the complex conjugate of the first two equations. We stress that these equations ensures the vanishing of the zero curvature conditions (2.14) for any value of $s$. For the case $s=1$ the states with spins \pm 2 do not appear, and we do not have the second equation in (1.1).

The first two equations in (1.1) can be obtained through another zero curvature equation. Consider the potentials [1]

$$
A_{\mu}^{(\alpha)}=\partial_{\mu} \alpha(u) Q \quad \tilde{B}_{\mu}^{(\beta)}=\beta(u) \mathcal{K}_{\mu} P
$$

where $\alpha$ and $\beta$ are functionals of $u$ only, and $Q$ and $P$ satisfy the Heisenberg algebra

$$
[Q, P]=\mathbb{1}
$$

The potential $A_{\mu}^{(\alpha)}$ is clearly flat, and $\tilde{B}_{\mu}^{(\beta)}$ does lie in an abelian ideal, generated by $P$ and $\mathbb{1}$. Then the zero curvature condition (2.14) gives

$$
D^{\mu} \tilde{B}_{\mu}^{(\beta)}=\left(\frac{\delta \beta}{\delta u} \partial^{\mu} u \mathcal{K}_{\mu}+\beta \partial^{\mu} \mathcal{K}_{\mu}\right) P+\beta \frac{\delta \alpha}{\delta u} \partial^{\mu} u \mathcal{K}_{\mu} \mathbb{1}
$$

Therefore, its vanishing implies the first two equations in (1.1). Replacing in (4.33), $\mathcal{K}_{\mu}$ by its complex conjugate, and $\alpha$ and $\beta$ by functions of $u^{*}$ only, one gets through (2.14) the corresponding complex conjugate equations.

Notice that we get more equations than the number of fields, and so we have a constrained system. However, there is a large class of vectors $\mathcal{K}_{\mu}$ for which the constraints are automatically satisfied. Consider the vector

$$
K_{\mu}=h_{\mu \nu} \partial^{\nu} u=\left(\partial^{\nu} u^{*} \partial_{\nu} u\right) \partial_{\mu} u-\left(\partial_{\nu} u\right)^{2} \partial_{\mu} u^{*}
$$

with $h_{\mu \nu}$ defined in (1.2).

Then, for any real function $\mathcal{F}\left(u, u^{*}, \partial u, \partial u^{*}\right)$

$$
\mathcal{K}_{\mu}=\mathcal{F} K_{\mu}
$$

satisfies identically the last two equations in (1.1). 
Therefore, for the class of vectors (4.37) the equations (1.1) reduce to the single complex equation

$$
\partial^{\mu}\left(\mathcal{F} K_{\mu}\right)=0
$$

Since (4.38) guarantees the zero curvature (2.14) for any value of the spin $s$ in the potential (4.32), and for any functional $\beta(u)$ in (4.33), it follows the model possesses an infinite number of conserved currents of the form (2.15). Notice that in the case of the potential (4.32) the infinity of the currents come from the infinite dimensional character of the abelian ideal, and in the case of (4.33) from the infinity of functionals $\beta(u)$. We will analyse these currents further in section 6 .

\section{Existence of Lagrangean}

The question then arises to know what are the models eq.(4.38) which can be derived from an action principle

$$
S=\int d^{d+1} x \mathcal{L}
$$

Quite generally $\mathcal{L}$ is a function which is Lorentz invariant and depends only on first order derivatives. So it is of the form

$$
\mathcal{L}=\mathcal{L}\left(\sigma, \sigma^{*}, \rho, u, u^{*}\right)
$$

where we define

$$
\sigma=\partial^{\mu} u \partial_{\mu} u, \quad \sigma^{*}=\partial^{\mu} u^{*} \partial_{\mu} u^{*}, \quad \rho=\partial^{\mu} u \partial_{\mu} u^{*}
$$

Then the equations of motion read

$$
\frac{\delta S}{\delta u}=\partial^{\mu}\left(-2 \frac{\partial \mathcal{L}}{\partial \sigma} \partial_{\mu} u-\frac{\partial \mathcal{L}}{\partial \rho} \partial_{\mu} u^{*}\right)+\frac{\partial \mathcal{L}}{\partial u}=0
$$

These equations of motion should be a linear combination of eq.(4.38) and its complex conjugate. Hence, we want to achieve

$$
\partial^{\mu}\left(-2 \frac{\partial \mathcal{L}}{\partial \sigma} \partial_{\mu} u-\frac{\partial \mathcal{L}}{\partial \rho} \partial_{\mu} u^{*}\right)+\frac{\partial \mathcal{L}}{\partial u}=\Lambda \partial^{\mu}\left(\mathcal{F} K_{\mu}\right)+\Phi \partial^{\mu}\left(\mathcal{F} K_{\mu}^{*}\right)
$$

where $\mathcal{L}, \mathcal{F}, \Lambda$ and $\Phi$ are functions to be determined. Moreover, $\mathcal{L}$ and $\mathcal{F}$ should be real. Denote

$$
T_{\mu \nu}=\partial_{\mu} u \partial_{\nu} u, \quad T_{\mu \nu}^{*}=\partial_{\mu} u^{*} \partial_{\nu} u^{*}, \quad S_{\mu \nu}=\partial_{\mu} u \partial_{\nu} u^{*}+\partial_{\mu} u^{*} \partial_{\nu} u
$$


The left hand side of eq.(5.39) becomes

$$
\begin{aligned}
& \left\{-4 \frac{\partial^{2} \mathcal{L}}{\partial \sigma^{2}} T_{\mu \nu}-\frac{\partial^{2} \mathcal{L}}{\partial \rho^{2}} T_{\mu \nu}^{*}-2 \frac{\partial^{2} \mathcal{L}}{\partial \sigma \partial \rho} S_{\mu \nu}-2 \frac{\partial \mathcal{L}}{\partial \sigma} \eta_{\mu \nu}\right\} \partial^{\mu \nu} u+ \\
& \left\{-2 \frac{\partial^{2} \mathcal{L}}{\partial \sigma \partial \rho} T_{\mu \nu}-2 \frac{\partial^{2} \mathcal{L}}{\partial \sigma^{*} \partial \rho} T_{\mu \nu}^{*}-\left(2 \frac{\partial^{2} \mathcal{L}}{\partial \sigma \partial \sigma^{*}}+\frac{1}{2} \frac{\partial^{2} \mathcal{L}}{\partial \rho^{2}}\right) S_{\mu \nu}-\frac{\partial \mathcal{L}}{\partial \rho} \eta_{\mu \nu}\right\} \partial^{\mu \nu} u^{*}+ \\
& -2 \sigma \frac{\partial^{2} \mathcal{L}}{\partial u \partial \sigma}-2 \rho \frac{\partial^{2} \mathcal{L}}{\partial u^{*} \partial \sigma}-\rho \frac{\partial^{2} \mathcal{L}}{\partial u \partial \rho}-\sigma^{*} \frac{\partial^{2} \mathcal{L}}{\partial u^{*} \partial \rho}+\frac{\partial \mathcal{L}}{\partial u}
\end{aligned}
$$

while the right hand side comes in two pieces

$$
\begin{aligned}
& \Lambda\left\{2 \rho \frac{\partial \mathcal{F}}{\partial \sigma} T_{\mu \nu}-\sigma \frac{\partial \mathcal{F}}{\partial \rho} T_{\mu \nu}^{*}-\left(\sigma \frac{\partial \mathcal{F}}{\partial \sigma}-\frac{\rho}{2} \frac{\partial \mathcal{F}}{\partial \rho}+\frac{1}{2} \mathcal{F}\right) S_{\mu \nu}+\rho \mathcal{F} \eta_{\mu \nu}\right\} \partial^{\mu \nu} u+ \\
& \Lambda\left\{\left(\rho \frac{\partial \mathcal{F}}{\partial \rho}+\mathcal{F}\right) T_{\mu \nu}-2 \sigma \frac{\partial \mathcal{F}}{\partial \sigma^{*}} T_{\mu \nu}^{*}+\left(\rho \frac{\partial \mathcal{F}}{\partial \sigma^{*}}-\frac{\sigma}{2} \frac{\partial \mathcal{F}}{\partial \rho}\right) S_{\mu \nu}-\sigma \mathcal{F} \eta_{\mu \nu}\right\} \partial^{\mu \nu} u^{*}+ \\
& \Lambda\left\{\frac{\partial \mathcal{F}}{\partial u^{*}}\left(\rho^{2}-\sigma \sigma^{*}\right)\right\}
\end{aligned}
$$

and

$$
\begin{aligned}
& \Phi\left\{-2 \sigma^{*} \frac{\partial \mathcal{F}}{\partial \sigma} T_{\mu \nu}+\left(\rho \frac{\partial \mathcal{F}}{\partial \rho}+\mathcal{F}\right) T_{\mu \nu}^{*}+\left(\rho \frac{\partial \mathcal{F}}{\partial \sigma}-\frac{\sigma^{*}}{2} \frac{\partial \mathcal{F}}{\partial \rho}\right) S_{\mu \nu}-\sigma^{*} \mathcal{F}^{*} \eta_{\mu \nu}\right\} \partial^{\mu \nu} u+ \\
& \Phi\left\{-\sigma^{*} \frac{\partial \mathcal{F}^{*}}{\partial \rho} T_{\mu \nu}+2 \rho \frac{\partial \mathcal{F}}{\partial \sigma^{*}} T_{\mu \nu}^{*}-\left(\sigma^{*} \frac{\partial \mathcal{F}}{\partial \sigma^{*}}-\frac{\rho}{2} \frac{\partial \mathcal{F}}{\partial \rho}+\frac{1}{2} \mathcal{F}\right) S_{\mu \nu}+\rho \mathcal{F} \eta_{\mu \nu}\right\} \partial^{\mu \nu} u^{*}+ \\
& \Phi\left\{\frac{\partial \mathcal{F}}{\partial u}\left(\rho^{2}-\sigma \sigma^{*}\right)\right\}
\end{aligned}
$$

Identification of the $\eta_{\mu \nu}$ terms gives:

$$
\begin{array}{rlrl}
\Lambda \mathcal{F} & =\frac{\alpha}{X}, & \alpha & \equiv \sigma^{*} \frac{\partial \mathcal{L}}{\partial \rho}+2 \rho \frac{\partial \mathcal{L}}{\partial \sigma} \\
\Phi \mathcal{F}=\frac{\beta}{X}, & \beta \equiv \rho \frac{\partial \mathcal{L}}{\partial \rho}+2 \sigma \frac{\partial \mathcal{L}}{\partial \sigma}
\end{array}
$$

where we set $X=\sigma \sigma^{*}-\rho^{2}$. Note that

$$
\rho \alpha-\sigma^{*} \beta=-2 X \frac{\partial \mathcal{L}}{\partial \sigma}, \quad-\sigma \alpha+\rho \beta=-X \frac{\partial \mathcal{L}}{\partial \rho}
$$

The functions $\Lambda$ and $\Phi$ can now be eliminated from the equations. For instance $\Lambda \frac{\partial \mathcal{F}}{\partial \rho}=$ $\frac{\alpha}{X} \frac{\partial \log \mathcal{F}}{\partial \rho}$ etc... From the $T_{\mu \nu}^{*} \partial^{\mu \nu} u$ term, we extract $\partial \log \mathcal{F} / \partial \rho$ :

$$
-X \frac{\partial \mathcal{L}}{\partial \rho} \frac{\partial \log \mathcal{F}}{\partial \rho}=-X \frac{\partial^{2} \mathcal{L}}{\partial \rho^{2}}-\beta
$$


Similarly, from the $S_{\mu \nu} \partial^{\mu \nu} u$ term we obtain $\partial \log \mathcal{F} / \partial \sigma$ :

$$
-X \frac{\partial \mathcal{L}}{\partial \rho} \frac{\partial \log \mathcal{F}}{\partial \sigma}=-X \frac{\partial^{2} \mathcal{L}}{\partial \sigma \partial \rho}+\alpha
$$

Finally from the $T_{\mu \nu}^{*} \partial^{\mu \nu} u^{*}$ term, we get $\partial \log \mathcal{F} / \partial \sigma^{*}$ :

$$
-X \frac{\partial \mathcal{L}}{\partial \rho} \frac{\partial \log \mathcal{F}}{\partial \sigma^{*}}=-X \frac{\partial^{2} \mathcal{L}}{\partial \sigma^{*} \partial \rho}
$$

Comparing eq.(5.42) and its complex conjugate, we find $\beta^{*}=\beta$ which from the definition of $\beta$ implies $\mathcal{L}=\mathcal{L}\left(\sigma \sigma^{*}, \rho, u, u^{*}\right)$. Similarly, comparing eq.(5.43) with the complex conjugate of eq. (5.44) we find $\alpha=0$, hence $\Lambda=0$, which means $\mathcal{L}=\mathcal{L}\left(X, u, u^{*}\right)$. With this information at hand, we find that the equations containing second order derivatives of $u$ reduce to

$$
\begin{aligned}
& \frac{\partial \mathcal{L}}{\partial X} \frac{\partial \log \mathcal{F}}{\partial \rho}=-2 \rho \frac{\partial^{2} \mathcal{L}}{\partial X^{2}} \\
& \frac{\partial \mathcal{L}}{\partial X} \frac{\partial \log \mathcal{F}}{\partial \sigma}=\sigma^{*} \frac{\partial^{2} \mathcal{L}}{\partial X^{2}} \\
& \frac{\partial \mathcal{L}}{\partial X} \frac{\partial \log \mathcal{F}}{\partial \sigma^{*}}=\sigma \frac{\partial^{2} \mathcal{L}}{\partial X^{2}}
\end{aligned}
$$

so that

$$
\mathcal{F}=f\left(u, u^{*}\right) \frac{\partial \mathcal{L}}{\partial X}, \quad \Longrightarrow \quad \Phi=\frac{2}{f}
$$

with $f\left(u, u^{*}\right)$ real. Finally eq.(5.39) reduces to

$$
\frac{\partial \mathcal{L}}{\partial u}=-2 X \frac{\partial \mathcal{L}}{\partial X} \frac{\partial \log f}{\partial u}
$$

which means that

$$
\mathcal{L}\left(X, u, u^{*}\right)=\mathcal{L}\left(\frac{X}{f^{2}\left(u, u^{*}\right)}\right)
$$

So the Lagrangean is a function of the square of the pull back of an area form $H$ on a two dimensional manifold

$$
H=\frac{1}{f} d u \wedge d u^{*}
$$

Let us summarize. Introduce

$$
h_{\mu \nu}=\partial_{\mu} u \partial_{\nu} u^{*}-\partial_{\nu} u \partial_{\mu} u^{*}
$$

The Lagrangean reads

$$
\mathcal{L}=\mathcal{L}\left(\frac{h^{2}}{2 f^{2}}\right), \quad h^{2}=h_{\mu \nu} h^{\mu \nu}
$$


The equations of motion are

$$
\partial^{\mu}\left(\frac{1}{f} \mathcal{L}^{\prime} K_{\mu}\right)=0, \quad K_{\mu}=h_{\mu \nu} \partial^{\nu} u
$$

or in expanded form:

$$
\frac{1}{2 f^{3}} \mathcal{L}^{\prime \prime} K_{\mu} \partial^{\mu} h^{2}+\frac{1}{f} \mathcal{L}^{\prime} \partial^{\mu} K_{\mu}-\frac{1}{f^{2}}\left(\frac{h^{2}}{f^{2}} \mathcal{L}^{\prime \prime}+\mathcal{L}^{\prime}\right) K_{\mu} \partial^{\mu} f=0
$$

Comparing (5.45) with (4.38) we see that for the models possessing Lagrangean we have $\mathcal{F} \equiv \mathcal{L}^{\prime} / f$. Therefore, the effect of imposing the existence of a Lagrangean restricts the classes of fuctionals $\mathcal{F}$ to those that depend on the derivatives of $u$ and $u^{*}$, only through $h^{2}$. The dependency on the fields is still quite general.

A special class of models models in a space-time of $d+1$ dimensions, is obtained by choosing

$$
\mathcal{L}=\left(\frac{h^{2}}{2 f^{2}}\right)^{d / 4}
$$

This has the advantage of circumventing Derrick's theorem, since the energy of static configurations will be invariant under scale transformations, $x^{\mu} \rightarrow \lambda x^{\mu}$ [2, 4 . The equations of motion read

$$
f\left((d-4) K_{\mu} \partial^{\mu} h^{2}+4 h^{2} \partial^{\mu} K_{\mu}\right)-2(d-2) h^{2} K_{\mu} \partial^{\mu} f=0
$$

or

$$
\mathcal{E} \equiv(d-4) h_{\mu \nu} \partial^{\nu} u \partial^{\mu} h^{2}+4 h^{2} \partial^{\mu} h_{\mu \nu} \partial^{\nu} u+(d-2)\left(h^{2}\right)^{2} \partial_{u^{*}} \log f=0
$$

Notice that for $d=2$ the equations of motion (and also the Lagrangean) do not depend upon $f$, and so on the determinant of the metric in target space. For $d=4$ the theory has the advantage of having a Lagrangean which is quadratic in time derivatives.

In order to have finite energy solutions one needs the field $u$ to go to a constant at spacial infinity. Therefore, the space $\mathbb{R}^{d}$ can be compactified on $S^{d}$, and so the finite energy solutions provide mappings from $S^{d}$ to the target space $U$, which are classified by the homotopy classes $\pi_{d}(U)$.

In the case where $U \equiv S^{2}$ we have $f=\left(1+u u^{*}\right)^{2}$ where $u$ and $u^{*}$ are the stereographic projection coordinates on $S^{2}$ :

$$
\vec{n}=\frac{1}{1+u u^{*}}\left(u+u^{*},-i\left(u-u^{*}\right),-1+u u^{*}\right), \quad \vec{n}^{2}=1, \quad u=\frac{n_{x}+i n_{y}}{1-n_{z}}
$$

Then we have that for $d=2$ and $d=3$ the homotopy groups are isomorphic to the integers under addition, i.e. $\pi_{2}\left(S^{2}\right)=\pi_{3}\left(S^{2}\right)=\mathbb{Z}$. In fact, for $d=2$ we have the winding number of $S^{2} \rightarrow S^{2}$, and for $d=3$ the Hopf invariant of $S^{3} \rightarrow S^{2}$. So, in such cases we can have solutions with non trivial topological charges. 


\section{Conserved currents}

Since our models have a zero curvature representation (2.14) with the potential (4.32) being valid in any spin $s$ representation, it follows they have an infinite number of conserved currents of the type (2.15). For any $s$, the current

$$
J_{\mu}^{(s)}=W^{-1} \tilde{B}_{\mu}^{(s)} W
$$

is conserved:

$$
\partial^{\mu} J_{\mu}^{(s)}=0
$$

Looking at eq. (4.32), we see that

$$
\tilde{B}_{\mu}^{(s)}=(\lambda \bar{\lambda})^{(s+1)} \tilde{B}_{\mu}^{(-1)}
$$

If we consider a general $\tilde{B}_{\mu}=\sum_{s} \beta_{s} \tilde{B}_{\mu}^{(s)}$, we have

$$
\tilde{B}_{\mu}=b(\lambda \bar{\lambda}) \tilde{B}_{\mu}^{(-1)}
$$

where $b(z)=\sum_{s} \beta_{s} z^{s+1}$ is essentially an arbitrary function.

Using (3.25) and (4.30) one can check that

$$
W^{-1} f(\lambda, \bar{\lambda}) W=f\left(\frac{\lambda-i u^{*} \bar{\lambda}}{\sqrt{1+|u|^{2}}}, \frac{\bar{\lambda}-i u \lambda}{\sqrt{1+|u|^{2}}}\right)
$$

Notice they look like a Lorentz boost on the space $(\lambda, \bar{\lambda})$ and with complex velocity $i u$. Indeed, the covering of the Lorentz group is $S L(2, \mathbb{C})$.

At the level of currents, this means

$$
J_{\mu}=b\left(\frac{\left(\lambda-i u^{*} \bar{\lambda}\right)(\bar{\lambda}-i u \lambda)}{1+u u^{*}}\right) J_{\mu}^{(-1)}
$$

We can write this in the nice form [2, 5]:

$$
J_{\mu}=\mathcal{K}_{\mu} \frac{\delta G}{\delta u}-\mathcal{K}_{\mu}^{*} \frac{\delta G}{\delta u *}
$$

where

$$
G=i \int^{v\left(u, u^{*}\right)} \frac{d v}{v^{2}} b(v), \quad v\left(u, u^{*}\right)=\frac{\left(\lambda-i u^{*} \bar{\lambda}\right)(\bar{\lambda}-i u \lambda)}{1+u u^{*}}
$$

For this, we have to check that

$$
\frac{\delta G}{\delta u}=\frac{1}{(\bar{\lambda}-i u \lambda)^{2}} b(v), \quad \frac{\delta G}{\delta u^{*}}=\frac{1}{\left(\lambda-i u^{*} \bar{\lambda}\right)^{2}} b(v)
$$


which follows easily from

$$
\frac{\delta v}{\delta u}=-i \frac{v^{2}}{(\bar{\lambda}-i u \lambda)^{2}}, \quad \frac{\delta v}{\delta u^{*}}=-i \frac{v^{2}}{\left(\lambda-i u^{*} \bar{\lambda}\right)^{2}}
$$

Notice the currents obtained in that way have the functional $G$ depending on $u$ and $u^{*}$. However, one can get "chiral" currents using the potential (4.33). Indeed the currents (2.15) for that potential are

$$
J_{\mu}^{(\beta)}=e^{\alpha Q} \tilde{B}_{\mu}^{(\beta)} e^{-\alpha Q}=\beta \mathcal{K}_{\mu} P+\alpha \beta \mathcal{K}_{\mu} \mathbb{1}
$$

and so they are of the form (6.50) with $G$ being a function of $u$ only.

Define

$$
\pi=\frac{\partial \mathcal{L}}{\partial \dot{u}}=\frac{1}{f^{2}} \mathcal{L}^{\prime} h_{0 \mu} \partial^{\mu} u^{*}=\frac{1}{f^{2}} \mathcal{L}^{\prime} K_{0}^{*}=\frac{1}{f} \mathcal{K}_{0}^{*}
$$

and impose the Poisson bracket

$$
\{\pi(x, t), u(y, t)\}=\delta(x-y)
$$

Then the conserved charges associate to $(6.50)$ become

$$
Q_{G}=i \int d^{3} x f\left(\pi^{*} \frac{\delta G}{\delta u}-\pi \frac{\delta G}{\delta u^{*}}\right)
$$

It is straightfoward to show that

$$
\left\{Q_{F}, Q_{G}\right\}=i Q_{\{F, G\}_{\text {Target }}}
$$

where

$$
\{F, G\}_{\text {Target }}=f\left(\frac{\delta F}{\delta u} \frac{\delta G}{\delta u^{*}}-\frac{\delta F}{\delta u^{*}} \frac{\delta G}{\delta u}\right)
$$

When the target space is $S^{2}$, this Poisson bracket is just the expression of Kirillov bracket $\left\{n_{i}, n_{j}\right\}_{S^{2}}=\epsilon_{i j k} n_{k}$ in the stereographic coordinates. From this we immediately get

$$
\left\{Q_{G}, u\right\}=-i f \partial_{u^{*}} G
$$

The meaning of the conserved currents is now clear: $Q_{G}$ generates area preserving diffeomorphisms [6]. Indeed, we are considering Lagrangeans of the form

$$
\mathcal{L}=\mathcal{L}\left(H_{\mu \nu} H^{\mu \nu}\right)
$$

where

$$
H=\frac{1}{f} h_{\mu \nu} d x^{\mu} \wedge d x^{\nu}=\frac{1}{f} d u \wedge d u^{*}
$$


The form $H$ is he pullback of an area form on target space. Therefore the action is invariant under area preserving diffeomorphisms. The conserved currents are the associated Noether currents.

The charge $Q_{G}$ generates the correct area preserving transformation for $u$ and $u^{*}$, when $G$ is real and a function of both $u$ and $u^{*}$. In the case of the diffeomorphisms coming from complex chiral functions depending on $u$ or $u^{*}$ only, the charge that generates the correct transformation under the Poisson bracket is a linear combination of charges of different chiralities, i.e.

$$
Q_{G}=i \int d^{3} x f\left(\pi^{*} \frac{\delta G(u)}{\delta u}-\pi \frac{\delta G^{*}\left(u^{*}\right)}{\delta u^{*}}\right)
$$

\section{$7 \quad$ Symmetries}

We now want to study the symmetries of the equations of motion, and not necessarily of the Lagrangean, of the models defined in (5.46). Extra symmetries, besides the area preserving diffeomorphisms, appear as we will show, due to the scaling properties of such models. We apply Sophus Lie theory as explained for instance in the book [7]. A careful analysis shows that the space-time and target space symmetries split into disjoint commuting sets. We shall then treat them separately.

\subsection{Space time symmetries.}

To say that $x^{\rho} \rightarrow x^{\rho}+\xi^{\rho}$ is a symmetry of a differential equation

$$
\mathcal{E}\left(x, u(x), \partial_{\mu} u(x), \partial_{\mu \nu} u(x)\right)=0
$$

where $\partial_{\mu \nu} \equiv \partial_{\mu} \partial_{\nu}$, means that if $u(x)$ is a solution so is $\tilde{u}(x) \equiv u(x-\xi)$, i.e.,

$$
\mathcal{E}\left(x, \tilde{u}(x), \partial_{\mu} \tilde{u}(x), \partial_{\mu \nu} \tilde{u}(x)\right)=0
$$

Now,

$$
\partial_{\mu} \tilde{u}(x)=\left(\partial_{\mu} u\right)(x-\xi)-\partial_{\mu} \xi^{\rho}(x)\left(\partial_{\rho} u\right)(x-\xi)
$$

and

$$
\begin{aligned}
\partial_{\mu \nu} \tilde{u}(x) & =\left(\partial_{\mu \nu} u\right)(x-\xi)-\partial_{\mu} \partial_{\nu} \xi^{\rho}(x)\left(\partial_{\rho} u\right)(x-\xi) \\
& -\partial_{\mu} \xi^{\rho}(x)\left(\partial_{\rho} \partial_{\nu} u\right)(x-\xi)-\partial_{\nu} \xi^{\rho}(x)\left(\partial_{\rho} \partial_{\mu} u\right)(x-\xi)
\end{aligned}
$$


Evaluating (7.52) at $x+\xi$ and keeping only linear terms in $\xi$, one gets that the vector field

$$
V=\sum_{\rho} \xi^{\rho} \partial_{\rho}
$$

generates a symmetry of the equations of motion if

$$
\delta \mathcal{E}\left(x, u, \partial_{\mu} u, \partial_{\mu \nu} u\right)=\Lambda_{1} \mathcal{E}+\Lambda_{2} \mathcal{E}^{*}
$$

with

$$
\begin{aligned}
\delta x^{\rho} & =\xi^{\rho} \\
\delta u & =0 \\
\delta \partial_{\mu} u & =-\partial_{\mu} \xi^{\nu} \partial_{\nu} u \\
\delta \partial_{\mu} \partial_{\nu} u & =-\left(\partial_{\mu} \partial_{\nu} \xi^{\rho}\right) \partial_{\rho} u-\partial_{\mu} \xi^{\rho} \partial_{\rho} \partial_{\nu} u-\partial_{\nu} \xi^{\rho} \partial_{\rho} \partial_{\mu} u
\end{aligned}
$$

Notice that the r.h.s. of (7.55) is taking into account the fact that we require the variation of the equation of motion to vanish only on the solution set of the complex equation $\mathcal{E}=0$.

Using this rule, one can evaluate the variations of all the quantities appearing in the equations of motion. We get for instance

$$
\begin{aligned}
\delta h_{\mu \nu} & =-\partial_{\mu} \xi^{\rho} h_{\rho \nu}-\partial_{\nu} \xi^{\rho} h_{\mu \rho} \\
\delta\left(\partial_{\sigma} h_{\mu \nu}\right) & =-\left(\partial_{\sigma} \partial_{\mu} \xi^{\rho}\right) h_{\rho \nu}-\left(\partial_{\sigma} \partial_{\nu} \xi^{\rho}\right) h_{\mu \rho}-\partial_{\sigma} \xi^{\rho} \partial_{\rho} h_{\mu \nu}-\partial_{\mu} \xi^{\rho} \partial_{\sigma} h_{\rho \nu}-\partial_{\nu} \xi^{\rho} \partial_{\sigma} h_{\mu \rho}
\end{aligned}
$$

It follows that

$$
\delta h^{2}=-2\left(\partial^{\mu} \xi^{\rho}+\partial^{\rho} \xi^{\mu}\right) h_{\rho \nu} h_{\mu}^{\nu}
$$

To have any chance for an invariance of the equations of motion, we require:

$$
\partial^{\mu} \xi^{\nu}+\partial^{\nu} \xi^{\mu}=2 D \eta_{\mathrm{eff}}^{\mu \nu}
$$

where $D$ is the common value of $\partial_{\mu} \xi^{\mu}$ (no summation). Notice that we have added a subscript eff. to the metric. The reason for it is that we may consider solutions of the equations of motion (5.47), which depend explicitly on the dimension $d+1$ of spacetime, but do not depend on some of the coordinates of space-time. That may happen for instance when one considers static solutions. In the above construction only the effective coordinates appear and we call the dimension and metric of that subspace as $d_{\text {eff. }}$ and $\eta_{\text {eff. }}^{\mu \nu}$ respectively. Then, we have

$$
\delta h^{2}=-4 D h^{2}
$$


and

$$
\delta \partial^{\mu} h_{\mu \nu}=-\left(\partial^{2} \xi^{\rho}\right) h_{\rho \nu}-2\left(\partial^{\mu} D\right) h_{\mu \nu}-2 D \partial^{\mu} h_{\mu \nu}-\partial_{\nu} \xi^{\rho} \partial^{\mu} h_{\mu \rho}
$$

but now

$$
\partial^{2} \xi^{\rho}=\partial_{\mu}\left(\partial^{\mu} \xi^{\rho}\right)=-\partial_{\mu} \partial^{\rho} \xi^{\mu}+2 \partial_{\mu} D \eta^{\mu \rho}=-\partial^{\rho} \partial_{\mu} \xi^{\mu}+2 \partial^{\rho} D=-\left(d_{\text {eff. }}-2\right) \partial^{\rho} D
$$

hence

$$
\delta \partial^{\mu} h_{\mu \nu}=\left(d_{\text {eff. }}-4\right) \partial^{\mu} D h_{\mu \nu}-2 D \partial^{\mu} h_{\mu \nu}-\partial_{\nu} \xi^{\rho} \partial^{\mu} h_{\mu \rho}
$$

From this, we quickly get

$$
\delta\left(h^{2} \partial^{\mu} h_{\mu \nu} \partial^{\nu} u\right)=-8 D h^{2} \partial^{\mu} h_{\mu \nu} \partial^{\nu} u+\left(d_{\text {eff. }}-4\right) h^{2}\left(\partial^{\mu} D\right) h_{\mu \nu} \partial^{\nu} u
$$

It is more cumbersome to obtain

$$
\delta\left(h_{\mu \nu} \partial^{\nu} u \partial^{\mu} h^{2}\right)=-8 D h_{\mu \nu} \partial^{\nu} u \partial^{\mu} h^{2}-4 h^{2}\left(\partial^{\mu} D\right) h_{\mu \nu} \partial^{\nu} u
$$

So for $\mathcal{E}$ given by eq.(5.47)

$$
\delta \mathcal{E}=-8 D \mathcal{E}-4\left(d-d_{\text {eff. }}\right) h^{2} \partial^{\mu} D h_{\mu \nu} \partial^{\nu} u
$$

Therefore, we conclude that

- If $d_{\text {eff. }}=d$, e.g. for static solutions, eq.(7.57) defines a symmetry of the equations of motion for all $D$.

- If $d_{\text {eff. }} \neq d$, eq.(7.57) defines a symmetry only if $\partial^{\mu} D=0$.

Eqs.(7.57) are not difficult to analyse. Consider it for $\mu \neq \nu$, and apply $\partial_{\mu} \partial_{\nu}$. Using that $D$ is the common value of $\partial_{\mu} \xi^{\mu}$ (no summation), we get that $\left(\partial^{\mu} \partial_{\mu}+\partial^{\nu} \partial_{\nu}\right) D=0$ for all pair of different indices. This implies that $\partial_{\mu}^{2} D=0$ for all $\mu$. Hence $D$ is at most linear in each of the variables $x^{\nu}$, and $\xi^{\mu}$ is at most quadratic. But then

$$
\partial_{\mu} \partial_{\nu} D=\partial_{\mu} \partial_{\nu}^{2} \xi^{\nu}=-\partial_{\nu}^{3} \xi^{\mu}=0, \quad \mu \neq \nu
$$

Hence $\partial_{\mu} \partial_{\nu} D=0, \forall \mu, \nu$. So $D$ is a linear function of the $x^{\nu}$. Then, we check that the set of vector fields $V^{(\mu)}$ (7.54) with components

$$
\xi^{(\mu) \nu}=x^{\mu} x^{\nu}-\frac{1}{2} \eta_{\text {eff. }}^{\mu \nu} x^{2}
$$

do satisfy the equations with $D^{(\mu)}=x^{\mu}$. This exhausts the solutions with $D$ strictly linear in $x^{\mu}$. If $D$ is a constant, the solution is

$$
\xi^{\nu}=x^{\nu}
$$

and corresponds to dilatations. Finally if $D=0$, we find the translations, rotations and Lorentz boosts (Poincaré group). When $d_{\text {eff. }} \neq d$ the transformations eq.(7.59) are excluded, and we are left only with Poincaré and dilatation symmetries. 


\subsection{Target space symmetries.}

This time the vector fields generating the symmetries act only on the target space, and the space-time coordinates are left unchanged, so

$$
\delta x^{\mu}=0
$$

and

$$
V=\Phi\left(u, u^{*}\right) \partial_{u}+\Phi^{*}\left(u, u^{*}\right) \partial_{u^{*}}
$$

Therefore, since $\delta$ and $\partial_{\mu}$ commute, one has

$$
\begin{aligned}
\delta u & =\Phi\left(u, u^{*}\right) \\
\delta \partial_{\mu} u & =\partial_{\mu} \Phi\left(u, u^{*}\right)=\partial_{u} \Phi \partial_{\mu} u+\partial_{u^{*}} \Phi \partial_{\mu} u^{*}
\end{aligned}
$$

and similarly for higher derivatives of $u$. Then

$$
\delta h_{\mu \nu}=\left(\partial_{u} \Phi+\partial_{u^{*}} \Phi^{*}\right) h_{\mu \nu}
$$

so that

$$
\delta h^{2}=2\left(\partial_{u} \Phi+\partial_{u^{*}} \Phi^{*}\right) h^{2}
$$

We find easily

$$
\begin{aligned}
\delta\left(h_{\mu \nu} \partial^{\nu} u \partial^{\mu} h^{2}\right) & =\left(4 \partial_{u} \Phi+3 \partial_{u^{*}} \Phi^{*}\right) h_{\mu \nu} \partial^{\nu} u \partial^{\mu} h^{2}+\partial_{u^{*}} \Phi h_{\mu \nu} \partial^{\nu} u^{*} \partial^{\mu} h^{2} \\
& -\partial_{u^{*}}\left(\partial_{u} \Phi+\partial_{u^{*}} \Phi^{*}\right)\left(h^{2}\right)^{2} \\
\delta\left(h^{2} \partial^{\mu} h_{\mu \nu} \partial^{\nu} u\right) & =\left(4 \partial_{u} \Phi+3 \partial_{u^{*}} \Phi^{*}\right) h^{2} \partial^{\mu} h_{\mu \nu} \partial^{\nu} u+\partial_{u^{*}} \Phi h^{2} \partial^{\mu} h_{\mu \nu} \partial^{\nu} u^{*} \\
& -\frac{1}{2} \partial_{u^{*}}\left(\partial_{u} \Phi+\partial_{u^{*}} \Phi^{*}\right)\left(h^{2}\right)^{2}
\end{aligned}
$$

Hence, again for $\mathcal{E}$ given by eq. (5.47), we find

$$
\delta \mathcal{E}=\left(4 \partial_{u} \Phi+3 \partial_{u^{*}} \Phi^{*}\right) \mathcal{E}-\partial_{u^{*}} \Phi \mathcal{E}^{*}-(d-2) Q\left(h^{2}\right)^{2}
$$

where

$$
\begin{aligned}
Q= & \partial_{u^{*}}\left(\partial_{u} \Phi+\partial_{u^{*}} \Phi^{*}\right)-\partial_{u} \log f \partial_{u^{*}} \Phi-\partial_{u^{*}} \log f \partial_{u^{*}} \Phi^{*} \\
& -\partial_{u} \partial_{u^{*}} \log f \Phi-\partial_{u^{*}} \partial_{u^{*}} \log f \Phi^{*}
\end{aligned}
$$

If $d=2$ we have a symmetry for any diffemorphism in target space. If $d \neq 2$, we have a symmetry only if $Q=0$. Setting, in that case,

$$
\Phi=f \widetilde{\Phi}
$$


the condition $Q=0$ becomes

$$
\partial_{u^{*}}\left(f\left[\partial_{u} \widetilde{\Phi}+\partial_{u^{*}} \widetilde{\Phi}^{*}\right]\right)=0
$$

So, the quantity $f\left[\partial_{u} \widetilde{\Phi}+\partial_{u^{*}} \widetilde{\Phi}^{*}\right]$ depends on $u$ only, but since it is real it must be a constant. We have to distinguish two cases. The first happens when that constant vanishes, and one has

$$
\partial_{u} \widetilde{\Phi}+\partial_{u^{*}} \widetilde{\Phi}^{*}=0
$$

Setting, for complex $F$ :

$$
\widetilde{\Phi}=\partial_{u^{*}} F
$$

the equation (7.63) becomes

$$
\partial_{u} \partial_{u^{*}}\left(F+F^{*}\right)=0
$$

Therefore, $F$ is either pure imaginary or $F$ is a function of $u^{*}$ only. These cases correspond to the area preserving diffeomorphisms.

The second case happens when the integration constant does not vanish (which we choose equals to 2 for convenience), and so

$$
\partial_{u} \widetilde{\Phi}+\partial_{u^{*}} \widetilde{\Phi}^{*}=\frac{2}{f}
$$

Setting $F$ real in (7.64) (since the imaginary part would lead to the area preserving diffeomorphisms discussed in the first case above) the equation (7.65) becomes

$$
\partial_{u} \partial_{u^{*}} F=\frac{1}{f}
$$

The function $F$ generates a new symmetry, not preserving the area:

$$
F=\int^{u} d u \int^{u^{*}} d u^{*} \frac{1}{f}
$$

Integration constants have to be chosen in order that $F\left(u, u^{*}\right)$ is real.

For the sphere, we have $f=\left(1+u u^{*}\right)^{2}$ and we find

$$
F=\log \left(1+u u^{*}\right), \quad \Longrightarrow \Phi=\left(1+u u^{*}\right) u
$$

For the plane, we have $f=1$ and we find

$$
F=u u^{*}, \quad \Longrightarrow \Phi=u
$$

and so, it is a scaling transformation on the target space (plane). 


\section{Solutions.}

Let us consider the equations of motion (5.47) in the static case. According to the analysis of section 7.1 we have $d_{\text {eff. }}=d$. The symmetries eq.(7.59) read

$$
x^{\prime i}=x^{i}+(\epsilon \cdot x) x^{i}-\frac{1}{2} x^{2} \epsilon^{i} \quad i=1,2, \ldots d
$$

They are conformal symmetries in the $d$-dimensional Euclidean space, because $d x^{\prime 2}=$ $(1+2 \epsilon \cdot x) d x^{2}$. These symmetries can be used to find static solutions to the equations of motion (5.47).

To do that, first recall the description of conformal symmetries of Euclidean space. Consider points of Euclidean space as spheres of radius zero. The equation for spheres is of the form (we use arrows to denote vectors in $d$-dimensional Euclidean space)

$$
\alpha \vec{x}^{2}-2 \vec{\beta} \cdot \vec{x}+\gamma=0
$$

or

$$
\left(\vec{x}-\frac{\vec{\beta}}{\alpha}\right)^{2}=\frac{\vec{\beta}^{2}-\alpha \gamma}{\alpha^{2}}
$$

So Euclidean space is described by

$$
\vec{\beta}^{2}-\alpha \gamma=0
$$

and

$$
\vec{x}=\frac{\vec{\beta}}{\alpha}
$$

Any linear transformation over the parameters $\alpha, \vec{\beta}$ and $\gamma$, which preserves eq.(8.68), acts on $\vec{x}$ through eq. (8.69). These are the conformal transformations. Write the quadratic expression (8.68) as

$$
\vec{\beta}^{2}-\alpha \gamma=\sum_{i=1}^{d} \beta_{i}^{2}+\left(\frac{a \alpha-a^{-1} \gamma}{2}\right)^{2}-\left(\frac{a \alpha+a^{-1} \gamma}{2}\right)^{2}
$$

where $a$ is an arbitrary scale. The group that leaves that quadractic form invariant is the conformal group $O(d+1,1)$. Denoting $d \equiv 2 n$ or $d=2 n-1$, according to $d$ being even or odd, we see that the maximum number of commuting, compact rotations is equal to $n$. In the case of $d$ odd, the first $n-1$ commuting rotations can be taken on the planes $\left(\beta_{2 j-1}, \beta_{2 j}\right)$, i.e.

$$
\delta \beta_{2 j-1}=\beta_{2 j}, \quad \delta \beta_{2 j}=-\beta_{2 j-1} \quad j=1,2, \ldots n-1
$$


The other one is then the rotation $\left(\beta_{2 n-1}, \frac{a \alpha-a^{-1} \gamma}{2}\right)$ :

$$
\delta \beta_{2 n-1}=\frac{a \alpha-a^{-1} \gamma}{2}, \quad \delta \frac{a \alpha-a^{-1} \gamma}{2}=-\beta_{2 n-1}, \quad \delta \frac{a \alpha+a^{-1} \gamma}{2}=0
$$

In the case of $d$ even, we have basically two distinct possibilities. In the first one we take $n$ commuting rotations as in (8.70), but with $j$ varying from 1 to $n$. In the second case we take the coordinate $\beta_{2 n}$ out of the game, and choose the $n$ commuting rotations as in the odd $d$ case, i.e. rotations (8.70) and (8.71).

Notice that when the rotations (8.70) are transported to $\vec{x}$-space using (8.69) one gets rotations on the planes $\left(x_{2 j-1}, x_{2 j}\right)$, i.e.

$$
\delta x_{2 j-1}=x_{2 j}, \quad \delta x_{2 j}=-x_{2 j-1} \quad j=1,2, \ldots n-1
$$

Now, let us transport the rotation (8.71) to $\vec{x}$-space. We have from (8.71) and (8.68)

$$
\delta \beta_{2 n-1}=\frac{a}{2} \alpha-\frac{a^{-1}}{2 \alpha} \vec{\beta}^{2}, \quad \delta \alpha=-a^{-1} \beta_{2 n-1}, \quad \delta \gamma=a \beta_{2 n-1}
$$

Of course, in the case of $d$ even the extra $\beta$ variable is left unchanged, i.e.

$$
\delta \beta_{2 n}=0
$$

Therefore for both cases, $d$ even or odd, we have that

$$
\begin{aligned}
\delta x_{i} & =a^{-1} \frac{\beta_{i} \beta_{2 n-1}}{\alpha^{2}}=\frac{x_{i} x_{2 n-1}}{a} \quad i \neq 2 n-1 \\
\delta x_{2 n-1} & =\frac{a}{2}-\frac{a^{-1}}{2} \frac{\vec{\beta}^{2}}{\alpha^{2}}+a^{-1} \frac{\beta_{2 n-1}^{2}}{\alpha^{2}}=\frac{1}{2 a}\left(x_{2 n-1}^{2}-\sum_{i \neq 2 n-1} x_{i}^{2}\right)+\frac{a}{2}
\end{aligned}
$$

We recover exactly one of our conformal transformations (8.67) with $\epsilon^{i}=\epsilon \delta_{i, 2 n-1}$, plus a translation in the $x_{2 n-1}$ direction.

Define vector fields corresponding to the rotations:

$$
\begin{aligned}
\partial_{\theta_{i}}=x_{2 i} \partial_{x_{2 i-1}}-x_{2 i-1} \partial_{x_{2 i}} \quad i=1,2, \ldots n-1 \\
\partial_{\theta_{n}}=\frac{x_{2 n-1}}{a} \sum_{i \neq 2 n-1} x_{i} \partial_{x_{i}}+\frac{1}{2 a}\left(a^{2}+x_{2 n-1}^{2}-\sum_{i \neq 2 n-1} x_{i}^{2}\right) \partial_{x_{2 n-1}}
\end{aligned}
$$

In the $u$ internal space we have a compact rotation which is the phase transformations of $u$, i.e. $u \rightarrow u e^{i \varphi}$. According to S. Lie, educated Ansatz for solving the equations of motion are obtained by imposing invariance under a combination of internal and external rotations, i.e. of the commuting vector fields

$$
\left[\partial_{\theta_{j}}-i m_{j}\left(u \partial_{u}-u^{*} \partial_{u^{*}}\right)\right] u=0 \quad j=1,2, \ldots n
$$


where $m_{j}$ are integer numbers. Then we have

$$
u=R\left(\zeta_{l}\right) e^{i \sum_{j=1}^{n} m_{j} \theta_{j}}
$$

where $\zeta_{l}$ are such that $\partial_{\theta_{j}} \zeta_{l}=0$, and are given as

$$
\zeta_{l}=\frac{a^{2}\left(x_{2 l-1}^{2}+x_{2 l}^{2}\right)}{\left(a^{2}+r^{2}\right)^{2}} \quad l=1,2, \ldots n-1
$$

where $r^{2} \equiv \sum_{i=1}^{d} x_{i}^{2}$. In the case of $d$ even, we have an extra $\zeta$ variable which is

$$
\zeta_{n}=\frac{a^{2} x_{2 n}^{2}}{\left(a^{2}+r^{2}\right)^{2}}
$$

For the other choice of commuting rotations in the case of $d$ even, namely the ones in (8.70) but with $j$ varying from 1 to $n$, the transformations in $\vec{x}$-space and corresponding vector fields are all of the form (8.72) and (8.73) respectively. Therefore, proceeding in the same manner we find the ansatz

$$
u=S\left(\rho_{l}\right) e^{i \sum_{j=1}^{n} m_{j} \theta_{j}}
$$

where $\partial_{\theta_{j}} \rho_{l}=0$, i.e.

$$
\rho_{l}=\frac{1}{a} \sqrt{x_{2 l-1}^{2}+x_{2 l}^{2}} \quad l=1,2, \ldots n
$$

The $n$ commuting conditions eqs. (8.75) introduce $n$ cyclic coordinates $\theta_{j}$, that will disappear from the equations of motion 1 . Hence, we know we will get a single differential equation for $R\left(\zeta_{l}\right)$ (and for $S\left(\rho_{l}\right)$ ). A priori $R\left(\zeta_{l}\right)$ and $S\left(\rho_{l}\right)$ are complex function. The form of the Ansatz is preserved if we perform an area preserving diffeomorphism depending only on $u u^{*}$. Indeed, from (6.51)

$$
\delta u=i f \partial_{u^{*}} G\left(u u^{*}\right)=i f G^{\prime}\left(u u^{*}\right) u
$$

Therefore, if $f$ does not depend upon the phase of $u$, like in the sphere where $f=$ $\left(1+u u^{*}\right)^{2}$, or on the plane where $f=1$, then change in $u$ is proportional to $u$ and the function multiplying it do not depend upon the phase of $u$. So, the form of the Ansatz is indeed preserved and

$$
\delta R=i\left(1+R R^{*}\right)^{2} G^{\prime}\left(R R^{*}\right) R
$$

\footnotetext{
${ }^{1}$ Remember that the variation of the equations of motion under a given symmetry implies $\delta \mathcal{E}=$ $\partial_{\theta_{j}} \mathcal{E}=\Lambda_{j} \mathcal{E}$. However, since the vector fields commute there must exist a $\chi$ such that $\Lambda_{j}=\partial_{\theta_{j}} \chi$ Then, $\mathcal{E}\left(\theta_{i}, \zeta_{l}\right)=\exp (\chi) \mathcal{E}\left(0, \zeta_{l}\right)$.
} 
This implies that

$$
\delta\left(R R^{*}\right)=0, \quad \text { and } \quad \delta\left(\frac{R}{R^{*}}\right)=2 i\left(1+R R^{*}\right)^{2} G^{\prime}\left(R R^{*}\right) \frac{R}{R^{*}}
$$

the real function $G$ can be used to kill one real function, for instance the phase of $R$. Hence, we can assume $R$ to be real. The same arguments apply to $S\left(\rho_{l}\right)$.

Let us particularize this construction for the two lowest dimensions. In the case $d=2$ we have that the static model is trivial. Indeed, its equation of motion (5.45) is given by

$$
\partial^{\mu}\left(\frac{h_{\mu \nu}}{\sqrt{h^{2}}} \partial^{\nu} u\right)=0
$$

In the static case $h_{\mu \nu}$ has just one component and so the above equation is satisfied by any configuration for the $u$ field.

In the case $d=3$ the coordinates $\zeta_{1} \equiv \zeta, \theta_{1} \equiv \varphi, \theta_{2} \equiv \xi$, are related to toroidal coordinates. Recall that the toroidal coordinates are defined as

$$
\begin{aligned}
& x=a q^{-1} \sinh \eta \cos \varphi \\
& y=a q^{-1} \sinh \eta \sin \varphi \\
& z=a q^{-1} \sin \xi \\
& q=\cosh \eta-\cos \xi
\end{aligned}
$$

Computing the vectors fields $\partial_{\varphi}$ and $\partial_{\xi}$ one recovers exactly (8.73) and (8.74) respectively. Moreover, we have

$$
\zeta=\frac{a^{2}\left(x^{2}+y^{2}\right)}{\left(a^{2}+x^{2}+y^{2}+z^{2}\right)^{2}}=\frac{1}{4} \tanh ^{2} \eta
$$

In the toroidal coordinates, the equation we get for $R(\eta)$ reads [2]

$$
\frac{\partial}{\partial \eta} \log \frac{R R^{\prime}}{\left(1+R^{2}\right)^{2}}=-\frac{2 m^{2} \sinh ^{2} \eta-n^{2} \cosh \eta}{m^{2} \sinh ^{2} \eta+n^{2} \sinh \eta}
$$

Imposing the boundary conditions (see (5.48))

$$
\begin{gathered}
\vec{n} \rightarrow(0,0,1) \quad \text { or } \quad|u| \rightarrow \infty \text { or } \quad R \rightarrow \infty \text { as } \eta \rightarrow 0 \\
\vec{n} \rightarrow(0,0,-1) \quad \text { or } \quad u \rightarrow 0 \quad \text { or } \quad R \rightarrow 0 \text { as } \eta \rightarrow \infty
\end{gathered}
$$

one gets

$$
u=R(\eta) e^{i(m \xi+n \theta)}, \quad R^{2}=\frac{\cosh \eta-\sqrt{n^{2} / m^{2}+\sinh ^{2} \eta}}{\sqrt{1+m^{2} / n^{2} \sinh ^{2} \eta}-\cosh \eta}
$$


The Hopf charge and the energy are

$$
Q_{H}=-n m, \quad E=(2 \pi)^{2} \sqrt{|n||m|(|n|+|m|)}
$$

The origin of the ansatz proposed in [2] to construct the hopfion solutions given above, is now clear: it comes from the conformal symmetry of the equations of motion in the 3-dimensional space. The toroidal coordinates appear because the two commuting conformal transformations coincide to rotations along the angles $\varphi$ and $\xi$.

\section{Extra Noether current}

The extra target space symmetry of the equations of motion, found in (7.66), is not a symmetry of the action. However, combining it with the space-time scaling transformation, one can find a symmetry of the action and so a Noether current. Although the procedure works for all Lagrangeans of the type (5.46) we will consider the $3+1$ dimensional model only $(d=3)$, on the sphere.

To compute the Noether currents, we shall use the old trick of making the parameter of the transformation to depend upon the space-time coordinates. Suppose a given action is invariant under a global transformation. If we make the parameter to depend on $x^{\mu}$ we should have that its variation is of the form

$$
\delta S=\int d^{4} x J^{\mu} \partial_{\mu} \varepsilon
$$

However, any variation of the action vanishes on shell. Hence, integrating by parts we get that the conservation law

$$
\partial_{\mu} J^{\mu}=0
$$

holds when the equations of motion are satisfied.

Under the target space transformation (7.66) we have that

$$
\delta\left(\frac{h^{2}}{2 f^{2}}\right)=2 \varepsilon f\left(\partial_{u} \widetilde{\Phi}+\partial_{u^{*}} \widetilde{\Phi}^{*}\right)\left(\frac{h^{2}}{2 f^{2}}\right)+\frac{2}{f} h^{\mu \nu}\left(\widetilde{\Phi} \partial_{\nu} u^{*}-\widetilde{\Phi}^{*} \partial_{\nu} u\right) \partial_{\mu} \varepsilon
$$

For the area preserving diffeomorphisms the $\epsilon$ term vanishes (see (7.63)). Then, one obtains the Noether current

$$
J_{\text {area pres. }}^{\mu}=\frac{3}{2 f}\left(\frac{h^{2}}{2 f^{2}}\right)^{-1 / 4} h^{\mu \nu}\left(\widetilde{\Phi} \partial_{\nu} u^{*}-\widetilde{\Phi}^{*} \partial_{\nu} u\right)
$$

which correspond to the currents 6.50 ). 
In the case of the extra transformation $(7.65)$ we get

$$
\delta\left(\frac{h^{2}}{2 f^{2}}\right)=4 \varepsilon\left(\frac{h^{2}}{2 f^{2}}\right)+\frac{2}{f} h^{\mu \nu}\left(\widetilde{\Phi} \partial_{\nu} u^{*}-\widetilde{\Phi}^{*} \partial_{\nu} u\right) \partial_{\mu} \varepsilon
$$

and so, for global transformation the action is not invariant, since $\delta S=3 \varepsilon S$.

To compensate for that non invariance we consider space-time scaling transformation of the form $x^{\prime \mu}=x^{\mu}-3 \varepsilon x^{\mu}$, where the factor -3 was introduced to compensate the factor 3 above. We have that

$$
\delta\left(d^{4} x\right)=-12 \varepsilon d^{4} x-3 x^{\nu} \partial_{\nu} \varepsilon d^{4} x
$$

Moreover from eq.(7.56) (or $(7.58)$ with $\xi^{\mu}=-3 \varepsilon x^{\mu}$, and so $D=-3$ ), we get:

$$
\delta h^{2}=12 \varepsilon h^{2}+12 x^{\rho} h_{\rho \nu} h^{\mu \nu} \partial_{\mu} \varepsilon
$$

The variation of the action coming from space dilatations is:

$$
\delta S=-3 \varepsilon S+\int d^{4} x\left(\frac{h^{2}}{2 f^{2}}\right)^{-1 / 4}\left(\frac{9}{2 f^{2}} x^{\rho} h_{\rho \nu} h^{\mu \nu}-3 x^{\mu} \frac{h^{2}}{2 f^{2}}\right) \partial_{\mu} \varepsilon
$$

Therefore, combining the two transformations as

$$
\delta u=\varepsilon \Phi, \quad \delta x^{\mu}=-3 \varepsilon x^{\mu}
$$

we get a symmetry of the action, and the corresponding Noether current is

$$
J^{\mu} \equiv-x^{\mu} \mathcal{L}+\left(\frac{h^{2}}{2 f^{2}}\right)^{-1 / 4} \frac{1}{2 f^{2}}\left(h^{\mu \nu}\left(\Phi \partial_{\nu} u^{*}-\Phi^{*} \partial_{\nu} u\right)+3 x^{\rho} h_{\rho \nu} h^{\mu \nu}\right)
$$

Recall that $\Phi$ is given at the end of section 7.2 for the cases of the sphere and plane.

There is a connection between this Noether current and the energy-momentum tensor. The canonical energy-momentum tensor is defined by

$$
\Theta_{\mu \nu}=\frac{\partial \mathcal{L}}{\partial \partial^{\mu} u} \partial_{\nu} u+\frac{\partial \mathcal{L}}{\partial \partial^{\mu} u^{*}} \partial_{\nu} u^{*}-g_{\mu \nu} \mathcal{L}
$$

and the conserved current 9.80 ) can be written as

$$
J_{\mu}=x^{\nu} \Theta_{\mu \nu}+j_{\mu}
$$

with

$$
j_{\mu} \equiv\left(\frac{h^{2}}{2 f^{2}}\right)^{-1 / 4} \frac{1}{2 f^{2}} h^{\mu \nu}\left(\Phi \partial_{\nu} u^{*}-\Phi^{*} \partial_{\nu} u\right)
$$


We now introduce the charge

$$
Q=\int d^{3} x J^{0}=\int d^{3} x\left(x^{\nu} \Theta_{0 \nu}+j_{0}\right)
$$

Then, for static configurations

$$
\frac{d Q}{d t}=-\int d^{3} x \mathcal{L}=E \equiv \text { static energy }=\int d \Sigma_{i} J_{i}
$$

From the above formula, the static energy of the toroidal solutions can be written as the flux of the current $\vec{J}$ through some boundary surface surrounding the singularities of the current $\vec{J}$. The field $u$ becomes singular when $\eta \rightarrow 0$ :

$$
u=C_{n, m} \frac{1}{\eta} e^{i m \xi+i n \varphi}, \quad C_{n, m}=|n|^{3 / 2} \sqrt{\frac{2}{|n||m|(|m|+|n|)}}
$$

From eq. (8.76) we have, when $\eta$ is small (we set $a=1$ )

$$
\eta=\frac{2 \rho}{1+r^{2}}, \quad q=\frac{2}{1+r^{2}}, \quad \sin \xi=\frac{2 z}{1+r^{2}}, \quad \tan \varphi=\frac{y}{x}
$$

from what we see that $\eta$ is small either when $\rho$ is small (z-axis) or $r$ is large (sphere at infinity).

One can check that the $x^{\nu} \Theta_{\mu \nu}$ part of (9.82) is regular, and that the $j_{\mu}$ part is regular at infinity. On the $z$-axis it behaves like

$$
\vec{j} \cdot \hat{e}_{\rho}=2 \sqrt{|n||m|(|m|+|n|)} \frac{1}{1+z^{2}} \frac{1}{\rho} \quad \rho \sim 0
$$

Therefore, the flux through an infinitesimally thin and infinite tube around the $z$-axis is obtained from the above expression by multiplying it by $\rho d \varphi d z$, and integrating over $\varphi$ and $z$, to get

$$
\text { flux }=(2 \pi)^{2} \sqrt{|n||m|(|m|+|n|)}=\text { static energy }
$$

We observe that the hopfion solutions carry a line structure with it, which is that line of singular flux of the conserved current (9.82).

\section{Conclusion.}

We have seen that the ideas of 国 to extend in higher dimensions some notions of integrability, do provide us with models with an infinite number of conserved currents. It turned out that these currents, in the case of the models discussed here, are associated to an invariance of the Lagrangean under area preserving diffeomorphisms. It 
is intriguing that they do not play an important role in the construction of the static solutions of the equations of motion. That role is played by the conformal symmetry of the static equations of motion, which leads to the construction of the relevant ansatz. It remains to see the role of the conserved currents in the scatterring processes of these solutions.

\section{Acknowledgements}

We are greatful to M. Bellon for discussions. We acknowledge the support of CAPES/COFECUB under the grant 306/00-II. LAF is partially supported by CNPq. 


\section{References}

[1] O. Alvarez, L.A. Ferreira, J. Sanchez Guillen, A new approach to integrable theories in any dimension. Nucl. Phys. B529 (1998) 689-736, hep-th/9710147

[2] H. Aratyn, L.A. Ferreira, A.H. Zimerman, Toroidal solitons in $3+1$ dimensional integrable theories., Phys. Lett. B456 (1999)162-170, hep-th/9902141.

Exact static soliton solutions of $3+1$ dimensional integrable theory with nonzero Hopf numbers. Phys. Rev. Lett. 83 (1999) 1723-1726, hep-th/9905079

[3] L. Breen and W. Messing, Differential Geometry of Gerbes, math.AG/0106083

R. Attal, Combinatorics of Non-Abelian Gerbes with Connection and Curvature, math-ph/0203056

J. C. Baez, Higher Yang-Mills Theory, hep-th/0206130.

[4] A. Kundu, Construction of non-linear sigma models in two and higher space dimensions, Ann. of Phys. 139 (1982) 36-47.

[5] K. Fujii, Y. Homma and T. Suzuki, Submodels of non-linear Grassmann sigma models in any dimension and conserved currents, exact solutions, Mod. Phys. Lett. A14 (1999) 919-928, hep-th/9809149.

[6] L.A. Ferreira, A.V. Razumov, Hopf solitons and area preserving diffeomorphisms of the sphere., Lett. Math. Phys. 55 (2001) 143-148, hep-th/0012176

[7] P. Olver, Applications of Lie groups to differential equations, 2nd edition, SpringerVerlag (1993). 